United Nations Educational Scientific and Cultural Organization and

International Atomic Energy Agency

THE ABDUS SALAM INTERNATIONAL CENTRE FOR THEORETICAL PHYSICS

\title{
GROUND STATE ENERGY OF A POLARON IN A SUPERLATTICE
}

\author{
S.Y. Mensah ${ }^{1}$ \\ Department of Physics, Laser and Fibre Optics Centre, University of Cape Coast, \\ Cape Coast, Ghana \\ and \\ The Abdus Salam International Centre for Theoretical Physics, Trieste, Italy, \\ F.K.A. Allotey \\ National Centre for Mathematical Sciences, Ghana Atomic Energy Commission, \\ Kwabenya, Accra, Ghana \\ and \\ The Abdus Salam International Centre for Theoretical Physics, Trieste, Italy, \\ G. Nkrumah \\ Department of Physics, University of Ghana, Legon Accra, Ghana \\ and \\ N.G. Mensah ${ }^{1}$ \\ Department of Mathematics, University of Cape Coast, Cape Coast, Ghana. \\ MIRAMARE - TRIESTE
}

October 2000

\footnotetext{
${ }^{1}$ Regular Associate of the Abdus Salam ICTP.
} 


\begin{abstract}
The ground state energy of a polaron in a superlattice was calculated using the doubletime Green functions. The effective mass of the polaron along the planes perpendicular to the superlattice axis was also calculated. The dependence of the ground state energy and the effective mass along the planes perpendicular to the superlattice axis on the electron-phonon coupling constant $\alpha$ and on the superlattice parameters (i.e. the superlattice period $d$ and the bandwidth $\Delta$ ) were studied. It was observed that if an infinite square well potential is assumed, the ground state energy of the polaron decreases (i.e. becomes more negative) with increasing $\alpha$ and $d$, but increases with increasing $\Delta$. For small values of $\alpha$, the polaron ground state energy varies slowly with $\Delta$, becoming approximately constant for large $\Delta$. The effective mass along the planes perpendicular to the superlattice axis was found to be approximately equal to the mass of an electron for all typical values of $\alpha, d$ and $\Delta$.
\end{abstract}




\section{Introduction}

Polarons in bulk materials have been extensively investigated for several years now, and many mathematical techniques have been used to solve the polaron problem. Mitra, Chatterjee, and Mukhopadhyay[1] and Peeters and Devreese[2] have given good reviews on polarons in bulk materials.

It is well known that when an electron moves slowly in a heterostructure such as superlattices (SLs), heterojunctions, dielectric slabs, quantum-wires, quantum-box, and quantum-wells (QWs) structures of polar crystals it may cause a distortion of the lattice, establishing a polarisation field which acts back on the electron, modifying its properties; in particular, the electron acquires a self-energy and an enhancement of its Bloch effective mass. The single electron, together with its accompanying distortion, is called a polaron. For example, some SLs, such as GaAs $/ \mathrm{Al}_{x} \mathrm{Ga}_{1-x} \mathrm{As}$ SLs are made up of polar compounds in which the interaction of an electron with optical phonons is an important mechanism that strongly influences the optical and transport properties of the SL.

Due to the presence of heterofaces in heterostructures, polaron effects in polar semiconductor heterostructures are very different from those in bulk materials, and the polaron problem in this case is much more complicated than the case in bulk materials. Hence approximate methods such as the Perturbation theory, the Lee-Low-Pines (LLP) Variational method[3], the Landau and Pekar Variational calculation, and the Green Function method are required for its solution.

A number of works have been done on the effects of polaron in heterostructures and we mention a few of them here. Lin, Chen and George[4] and Hai, Peeters and Devreese[5] investigated the electron-phonon interaction and the polaron states in a symmetric single QW. Hai, Peeters and Devreese [6] further studied magnetopolaron in a GaAs/AlAs symmetric QW. Polaron in a symmetric single QW within an electric field has been investigated by Chen, Liang and Li[7]. Shi, Zhu, Liu, Pan and Li[8] investigated the ground state polaron energy and the effective mass in asymmetric single and step QWs by using the second-order Perturbation theory and the modified LPP Variational method. Magnetopolarons in quantum dots were investigated in [9]. Zhou and $\mathrm{Gu}[10]$ investigated magnetopolarons in cylindrical quantum wires. Magnetopolaron in a QW has been studied in the case of a weak external magnetic field in [11]. Haupt and Wendler[12] studied the resonant magnetopolaron effects in parabolic QWs. Magnetopolarons in a heterojunction were investigated by means of the Green function method in [13] and [14]. Licari[15], and Liang, Gu and Lin[16] studied the polaron states in a polar slab using secondorder Perturbation theory. The self-trapping energy of a polaron in a polar-crystal slab in a magnetic field has been obtained by Wei, Zhao, and Gu[17] using the Larsen Perturbation theory. $\mathrm{Lu}$ and $\mathrm{Li}[18]$ studied the properties of a polaron in a polar slab. The polaron effects in a heterojunction were investigated by Degani and Hipólito[19]. The interface polaron in a heterojunction in a magnetic field was investigated by Ban, Liang and Zheng[20]. Farias, Degani and 
Hipólito[21] studied bound polarons in a heterojunction. Surface polarons in a bilayer system are given in [22]. Thilagam and Singh [23] investigated, in the infinite-square-well approximation, polarons in quasi-two dimensional structures in which only confined bulklike longitudinal optical(LO) phonons were considered. Also Zheng, Ban and Liang [24], studied confined bulklike LO and interface phonons, and investigated the properties of a polaron in an infinite QW. Using the Landau-Pekar theory, a strong-coupling theory of quasi-two dimensional polarons, in which the contribution of the interface modes to the polaron effect is ignored, was proposed in [25]. The binding energies of bound polarons in strong magnetic fields in a QW, a quantum-well wire and a quantum box were given in [26]. Zhou and $\mathrm{Gu}[27]$ also studied impurity bound polarons in a QW. Bound magnetic polarons in a QW were studied in [28].

Though quite a number of works have been done on polarons in bulk materials and heterostructures, to the best of our knowledge, little work has been done on polaron effects in SLs in particular. In our present work, we shall calculate the ground state energy and the effective mass of a polaron in SL, using the Double Time Green Functions. This approach provides a relatively easier and convenient way of calculating the shift in the ground state energy of a polaron formed as a result of electron-phonon interaction in a superlattice.

In this paper it will be indicated that the ground state energy of the polaron depends on $d$ and $\Delta$, if an infinite square well potential is assumed. The ground state energy of the polaron will be shown to decrease (becomes more negative) with increasing $\alpha$ and $d$, but increases with increasing $\Delta$. For small values of $\alpha$, the polaron ground state energy varies slowly with $\Delta$, becoming approximately constant for large $\Delta$. On the other hand, the effective mass along the plane perpendicular to the SL axis will be found to be approximately equal to the mass of an electron for all typical values of $\alpha, d$ and $\Delta$.

The present work is organised as follows. In sections 2 and 3, we present the theory and the calculation of the shift in the ground state energy of a polaron in a superlattice. The results and its discussion are presented in section 4 . The conclusion is given in section 5 .

\section{Theory}

The Hamiltonian for an electron-phonon interaction in the second quantization formalism is

$$
\begin{aligned}
H & =H_{o}+H_{i n t} \\
\text { with } H_{o} & =\sum_{\boldsymbol{p}, s} \varepsilon_{\boldsymbol{p}}^{s} a_{\boldsymbol{p}}^{s \dagger} a_{\boldsymbol{p}}^{s}+\sum_{\boldsymbol{q}} \omega_{\boldsymbol{q}} b_{\boldsymbol{q}}^{\dagger} b_{\boldsymbol{q}} \\
H_{\text {int }} & =\sum_{\boldsymbol{p} \boldsymbol{p}^{\prime}} \sum_{\boldsymbol{q} s s^{\prime}}\left\langle s, \boldsymbol{p}|V(\boldsymbol{r})| s^{\prime}, \boldsymbol{p}^{\prime}\right\rangle a_{\boldsymbol{p}}^{s \dagger} a_{\boldsymbol{p}^{\prime}}^{s^{\prime}}\left(b_{\boldsymbol{q}}+b_{-\boldsymbol{q}}^{\dagger}\right) \\
\varepsilon_{\boldsymbol{p}}^{s} & =\frac{p_{\perp}^{2}}{2 m}+\varepsilon_{s}-\Delta_{s} \cos \left(p_{z} d\right), s=1,2, \ldots \\
p_{\perp}^{2} & =p_{x}^{2}+p_{y}^{2}
\end{aligned}
$$


$\varepsilon_{s}$ and $\Delta_{s}$ are determined by SL parameters[29]; $p_{z}$ and $p_{\perp}$ are respectively, the components of the crystal momentum along and perpendicular to the SL axis; $d$ is the SL period, $a_{\boldsymbol{p}}^{s \dagger}, a_{\boldsymbol{p}}^{s}$ are the creation and the annihilation operators of an electron in the $s$-th miniband; $\omega_{\boldsymbol{q}}$ is the phonon frequency with wave vector $\boldsymbol{q}$; and $b_{\boldsymbol{q}}^{\dagger}, b_{\boldsymbol{q}}$ are the phonon creation and annihilation operators.

The matrix element in Eqn.(3) is given in [30] as

$$
\begin{aligned}
\left\langle s, \boldsymbol{p}|V(\boldsymbol{r})| s^{\prime}, \boldsymbol{p}^{\prime}\right\rangle & =\int \psi_{\boldsymbol{p}}^{s *}(\boldsymbol{r}) V(\boldsymbol{r}) \psi_{\boldsymbol{p}^{\prime}}^{s^{\prime}}(\boldsymbol{r}) d \boldsymbol{r} \\
\text { where } \psi_{\boldsymbol{p}}^{s} & =\frac{1}{L} \exp \left[i\left(p_{x} x+p_{y} y\right)\right] \frac{1}{N} \sum_{j} \psi_{s}(z-f d) \exp \left(i p_{z} f d\right)
\end{aligned}
$$

$L$ is the normalized length, $N$ is the number of periods in the SL. $\psi_{s}(z)$ is the wave function of an electron in the $s$-th state in one of the one dimensional potential wells which composes the SL potential. In particular, the matrix element for a scattering potential $V(\boldsymbol{r})$ is

$$
\left\langle s, \boldsymbol{p}|V(\boldsymbol{r})| s^{\prime}, \boldsymbol{p}^{\prime}\right\rangle=\sum_{n=-\infty}^{\infty} C_{\boldsymbol{q}} M_{s s^{\prime}}\left(q_{z}\right) \delta_{q_{x}, p_{x}-p_{x}^{\prime}} \delta_{q_{y}, p_{y}-p_{y}^{\prime}} \delta_{q_{z}, p_{z}-p_{z}^{\prime}+n \boldsymbol{g}}
$$

where $\boldsymbol{g}=(0,0,2 \pi / d)$ is a reciprocal SL vector. $C_{\boldsymbol{q}}$ are the Fourier components of $V(\boldsymbol{r})$ and

$$
M_{s s^{\prime}}\left(q_{z}\right)=\int \psi_{s}^{*}(z) \psi_{s^{\prime}}(z) e^{i q_{z} z} d z
$$

Using Eqns.(8) and (9), Eqn.(3) can be written as

$$
H_{i n t}=\sum_{\boldsymbol{p} \boldsymbol{q} n} \sum_{s s^{\prime}} C_{\boldsymbol{q}} M_{s s^{\prime}}\left(q_{z}\right) a_{\boldsymbol{p}}^{s^{\dagger}} a_{\boldsymbol{p}-\boldsymbol{q}+n \boldsymbol{g}}^{s^{\prime}}\left(b_{\boldsymbol{q}}+b_{-\boldsymbol{q}}^{\dagger}\right)
$$

In order to obtain the dispersion relation for electrons we write the equations of motion for $a_{\boldsymbol{p}}^{s \dagger}, a_{\boldsymbol{p}}^{s}, b_{\boldsymbol{q}}^{\dagger}, b_{\boldsymbol{q}}$.

$$
\begin{aligned}
i \frac{d a_{\boldsymbol{p}}^{s}}{d t} & =\varepsilon_{\boldsymbol{p}}^{s} a_{\boldsymbol{p}}^{s}+\sum_{\boldsymbol{q} n s^{\prime}} C_{\boldsymbol{q}} M_{s s^{\prime}}\left(q_{z}\right) a_{\boldsymbol{p}-\boldsymbol{q}+n \boldsymbol{g}}^{s^{\prime}}\left(b_{\boldsymbol{q}}+b_{-\boldsymbol{q}}^{\dagger}\right) \\
-i \frac{d a_{\boldsymbol{p}}^{s \dagger}}{d t} & =\varepsilon_{\boldsymbol{p}}^{s} a_{\boldsymbol{p}}^{s \dagger}+\sum_{\boldsymbol{q} n s^{\prime}} C_{\boldsymbol{q}} M_{s s^{\prime}}\left(q_{z}\right) a_{\boldsymbol{p}+\boldsymbol{q}-n \boldsymbol{g}}^{s^{\prime} \dagger}\left(b_{\boldsymbol{q}}+b_{-\boldsymbol{q}}^{\dagger}\right) \\
i \frac{d b_{\boldsymbol{q}}}{d t} & =\omega_{\boldsymbol{q}} b_{\boldsymbol{q}}+\sum_{\boldsymbol{p} n s s^{\prime}} C_{\boldsymbol{q}} M_{s s^{\prime}}\left(-q_{z}\right) a_{\boldsymbol{p}-\boldsymbol{q}-n \boldsymbol{g}}^{s \dagger} a_{\boldsymbol{p}}^{s^{\prime}} \\
-i \frac{d b_{-\boldsymbol{q}}^{\dagger}}{d t} & =\omega_{\boldsymbol{q}} b_{-\boldsymbol{q}}^{\dagger}+\sum_{\boldsymbol{p} n s s^{\prime}} C_{\boldsymbol{q}} M_{s s^{\prime}}\left(-q_{z}\right) a_{\boldsymbol{p}-\boldsymbol{q}-n \boldsymbol{g}}^{\dagger} a_{\boldsymbol{p}}^{s^{\prime}}
\end{aligned}
$$

The single particle Green functions of the electron type $G_{\boldsymbol{p}}\left(t-t^{\prime}\right)$ are defined as

$$
G_{\boldsymbol{p}}\left(t-t^{\prime}\right)=-i \theta\left(t-t^{\prime}\right)\left\langle\left\{a_{\boldsymbol{p}}^{s}(t), a_{\boldsymbol{p}}^{s^{\prime} \dagger}\left(t^{\prime}\right)\right\}\right\rangle=\left\langle\left\langle a_{\boldsymbol{p}}^{s}(t) ; a_{\boldsymbol{p}}^{s^{\prime} \dagger}\left(t^{\prime}\right)\right\rangle\right\rangle
$$

where $\{A, B\}$ is the anticommutator of $A$ and $B$,

$$
\{A, B\}=A B+B A
$$


and

$$
\theta\left(t-t^{\prime}\right)= \begin{cases}1 & t>t^{\prime} \\ 0 & t<t^{\prime}\end{cases}
$$

is called the Heaviside step function. Using Eqns.(11)-(14) the following equation of motion for $G_{\boldsymbol{p}}\left(t-t^{\prime}\right)$ is obtained,

$$
\begin{aligned}
i \frac{d G_{\boldsymbol{p}}\left(t-t^{\prime}\right)}{d t}= & \delta\left(t-t^{\prime}\right)+G_{\boldsymbol{p}}\left(t-t^{\prime}\right)+\sum_{\boldsymbol{q} n l} C_{\boldsymbol{q}} M_{s l}\left(q_{z}\right) \\
& \cdot\left(\Gamma_{\boldsymbol{p}-\boldsymbol{q}, \boldsymbol{p}, \boldsymbol{q}}\left(t-t^{\prime}\right)+\Gamma_{\boldsymbol{p}-\boldsymbol{q}, \boldsymbol{p}, \boldsymbol{q}}^{\prime}\left(t-t^{\prime}\right)\right)
\end{aligned}
$$

where $\Gamma_{\boldsymbol{p}-\boldsymbol{q}, \boldsymbol{p}, \boldsymbol{q}}$ and $\Gamma_{\boldsymbol{p}-\boldsymbol{q}, \boldsymbol{p}, \boldsymbol{q}}^{\prime}$ are mixed types of Green functions defined respectively as

$$
\begin{aligned}
\Gamma_{\boldsymbol{p}-\boldsymbol{q}, \boldsymbol{p}, \boldsymbol{q}}\left(t-t^{\prime}\right) & =\left\langle\left\langle\left. a_{\boldsymbol{p}-\boldsymbol{q}+n \boldsymbol{g}}^{l} b_{\boldsymbol{q}}\right|_{t} ; a_{\boldsymbol{p}}^{s^{\prime} \dagger}\left(t^{\prime}\right)\right\rangle\right\rangle \\
\Gamma_{\boldsymbol{p}-\boldsymbol{q}, \boldsymbol{p}, \boldsymbol{q}}^{\prime}\left(t-t^{\prime}\right) & =\left\langle\left\langle\left. a_{\boldsymbol{p}-\boldsymbol{q}+n \boldsymbol{g}}^{l} b_{-\boldsymbol{q}}^{\dagger}\right|_{t} ; a_{\boldsymbol{p}}^{s^{\prime} \dagger}\left(t^{\prime}\right)\right\rangle\right\rangle
\end{aligned}
$$

By constructing equations of motion for $\Gamma_{\boldsymbol{p}-\boldsymbol{q}, \boldsymbol{p}, \boldsymbol{q}}$ and $\Gamma_{\boldsymbol{p}-\boldsymbol{q}, \boldsymbol{p}, \boldsymbol{q}}^{\prime}$ and decoupling the higher order Green functions occurring in these equations, we obtain the following equations for $\Gamma_{\boldsymbol{p}-\boldsymbol{q}, \boldsymbol{p}, \boldsymbol{q}}$ and $\Gamma_{\boldsymbol{p}-\boldsymbol{q}, \boldsymbol{p}, \boldsymbol{q}}^{\prime}$ respectively:

$$
\begin{aligned}
i \frac{d \Gamma_{\boldsymbol{p}-\boldsymbol{q}, \boldsymbol{p}, \boldsymbol{q}}\left(t-t^{\prime}\right)}{d t}= & \left(\varepsilon_{\boldsymbol{p}-\boldsymbol{q}}^{s}+\omega_{\boldsymbol{q}}\right) \Gamma_{\boldsymbol{p}-\boldsymbol{q}, \boldsymbol{p}, \boldsymbol{q}} \\
& +C_{\boldsymbol{q}} M_{s s}\left(q_{z}\right)\left(N_{\boldsymbol{q}}+1-f_{\boldsymbol{p}-\boldsymbol{q}}^{s}\right) G_{\boldsymbol{p}}\left(t-t^{\prime}\right) \\
i \frac{d \Gamma_{\boldsymbol{p}-\boldsymbol{q}, \boldsymbol{p}, \boldsymbol{q}}^{\prime}\left(t-t^{\prime}\right)}{d t}= & \left(\varepsilon_{\boldsymbol{p}-\boldsymbol{q}}^{s}-\omega_{\boldsymbol{q}}\right) \Gamma_{\boldsymbol{p}-\boldsymbol{q}, \boldsymbol{p}, \boldsymbol{q}}^{\prime} \\
& +C_{\boldsymbol{q}} M_{s s}\left(q_{z}\right)\left(f_{\boldsymbol{p}-\boldsymbol{q}}^{s}+N_{\boldsymbol{q}}\right) G_{\boldsymbol{p}}\left(t-t^{\prime}\right)
\end{aligned}
$$

where $N_{\boldsymbol{q}}=\left\langle b_{\boldsymbol{q}}^{\dagger} b_{\boldsymbol{q}}\right\rangle$ and $f_{\boldsymbol{p}-\boldsymbol{p}}^{s}=\left\langle a_{\boldsymbol{p}-\boldsymbol{q}}^{s \dagger} a_{\boldsymbol{p}-\boldsymbol{q}}^{s}\right\rangle$ are the number density of phonons and electrons respectively.

Writing Eqns.(18), (21) and (22) in terms of Fourier components of the Green functions, with

$$
\begin{aligned}
G_{\boldsymbol{p}}(t) & =\int_{-\infty}^{\infty} G_{\boldsymbol{p}}(E) e^{-i E t} d t \\
\Gamma_{\boldsymbol{p}-\boldsymbol{q}, \boldsymbol{p}, \boldsymbol{q}}(t) & =\int_{-\infty}^{\infty} \Gamma_{\boldsymbol{p}-\boldsymbol{q}, \boldsymbol{p}, \boldsymbol{q}}(E) e^{-i E t} d t \\
\Gamma_{\boldsymbol{p}-\boldsymbol{q}, \boldsymbol{p}, \boldsymbol{q}}^{\prime}(t) & =\int_{-\infty}^{\infty} \Gamma_{\boldsymbol{p}-\boldsymbol{q}, \boldsymbol{p}, \boldsymbol{q}}^{\prime}(E) e^{-i E t} d t \\
\delta(t) & =\frac{1}{2 \pi} \int_{-\infty}^{\infty} e^{-i E t} d t,
\end{aligned}
$$

one finds

$$
\left(E-\varepsilon_{\boldsymbol{p}}^{s}\right) G_{\boldsymbol{p}}(E)=\frac{1}{2 \pi}+\sum_{\boldsymbol{q} n s} C_{\boldsymbol{q}} M_{s s}\left(q_{z}\right)\left(\Gamma_{\boldsymbol{p}-\boldsymbol{q}, \boldsymbol{p}, \boldsymbol{q}}(E)+\Gamma_{\boldsymbol{p}-\boldsymbol{q}, \boldsymbol{p}, \boldsymbol{q}}^{\prime}(E)\right)
$$


where

$$
\begin{aligned}
\Gamma_{\boldsymbol{p}-\boldsymbol{q}, \boldsymbol{p}, \boldsymbol{q}}(E) & =\frac{C_{\boldsymbol{q}} M_{s s}(q)\left(N_{\boldsymbol{q}}+1-f_{\boldsymbol{p}-\boldsymbol{q}}^{s}\right) G_{\boldsymbol{p}}(E)}{E-\varepsilon_{\boldsymbol{p}-\boldsymbol{q}}^{s}-\omega_{\boldsymbol{q}}} \\
\Gamma_{\boldsymbol{p}-\boldsymbol{q}, \boldsymbol{p}, \boldsymbol{q}}^{\prime}(E) & =\frac{C_{\boldsymbol{q}} M_{s s}\left(q_{z}\right)\left(f_{\boldsymbol{p}-\boldsymbol{q}}^{s}+N_{\boldsymbol{q}}\right) G_{\boldsymbol{p}}(E)}{E-\varepsilon_{\boldsymbol{p}-\boldsymbol{q}}^{s}+\omega_{\boldsymbol{q}}}
\end{aligned}
$$

and hence

$$
G_{\boldsymbol{p}}(E)=\frac{1}{2 \pi\left(E-\varepsilon_{\boldsymbol{p}}^{s}-M_{\boldsymbol{p}}(E)\right)}
$$

where

$$
M_{\boldsymbol{p}}(E)=\sum_{\boldsymbol{q} s}\left|C_{\boldsymbol{q}} M_{s s}\left(q_{z}\right)\right|^{2}\left(\frac{N_{\boldsymbol{q}}+1-f_{\boldsymbol{p}-\boldsymbol{q}}^{s}}{E-\varepsilon_{\boldsymbol{p}-\boldsymbol{q}}^{s}-\omega_{\boldsymbol{q}}}+\frac{N_{\boldsymbol{q}}+f_{\boldsymbol{p}-\boldsymbol{q}}^{s}}{E-\varepsilon_{\boldsymbol{p}-\boldsymbol{q}}^{s}+\omega_{\boldsymbol{q}}}\right)
$$

is called the mass operator. Eqn.(30) is the dispersion equation. Assuming the band gaps are to be wide such that there are no interband transitions, the band label $s$ can be dropped.

Green functions can also be used to obtain the correlation functions[31],

$$
\left\langle a_{\boldsymbol{p}}^{\dagger}\left(t^{\prime}\right) ; a_{\boldsymbol{p}}(t)\right\rangle=\int_{-\infty}^{\infty} J_{\boldsymbol{p}}(\omega) e^{-i \omega\left(t-t^{\prime}\right)} d \omega
$$

where $J_{p}$ is the spectral intensity given by

$$
G_{\boldsymbol{p}}(\omega+i \epsilon)-G_{\boldsymbol{p}}(\omega-i \epsilon)=-J_{\boldsymbol{p}}(\omega)\left(e^{\beta \omega}+1\right)
$$

Inserting Eqn.(30) into Eqn.(32), we obtain

$$
M_{\boldsymbol{p}}(\omega \pm i \epsilon)=M_{\boldsymbol{p}}(\omega) \mp i \gamma_{\boldsymbol{p}}(\omega)
$$

where

$$
\begin{aligned}
\gamma_{\boldsymbol{p}}(\omega)= & \pi \sum_{\boldsymbol{q}}\left|C_{\boldsymbol{q}} M\left(q_{z}\right)\right|^{2}\left\{\left(N_{\boldsymbol{q}}+1-f_{\boldsymbol{p}-\boldsymbol{q}}\right) \delta\left(\omega-\varepsilon_{\boldsymbol{p}-\boldsymbol{q}}-\omega_{\boldsymbol{q}}\right)+\right. \\
& \left.+\left(f_{\boldsymbol{p}-\boldsymbol{q}}+N_{\boldsymbol{q}}\right) \delta\left(\omega-\varepsilon_{\boldsymbol{p}-\boldsymbol{q}}+\omega_{\boldsymbol{q}}\right)\right\}
\end{aligned}
$$

is the coefficient of absorption for electrons.

In order to obtain the shift in the ground state energy of an electron as a result of its interaction with phonons, the mass operator $M_{\boldsymbol{p}}(\omega)$ is expanded in a power series in $\omega$ at $\omega=\bar{\varepsilon}_{\boldsymbol{p}}$, taking into account that $\gamma_{\boldsymbol{p}}(\omega)$ is a slowly varying function, i.e. $\gamma_{\boldsymbol{p}}(\omega) \approx \gamma_{\boldsymbol{p}}\left(\bar{\varepsilon}_{\boldsymbol{p}}\right)$. This implies that

$$
\bar{\varepsilon}_{\boldsymbol{p}}-\varepsilon_{\boldsymbol{p}}-M_{\boldsymbol{p}}\left(\bar{\varepsilon}_{\boldsymbol{p}}\right)=0
$$

i.e.

$$
\bar{\varepsilon}_{\boldsymbol{p}}-\varepsilon_{\boldsymbol{p}}-P \sum_{\boldsymbol{q}}\left|C_{\boldsymbol{q}} M\left(q_{z}\right)\right|^{2}\left(\frac{N_{\boldsymbol{q}}+1-f_{\boldsymbol{p}-\boldsymbol{q}}}{\bar{\varepsilon}_{\boldsymbol{p}}^{o}-\bar{\varepsilon}_{\boldsymbol{p}-\boldsymbol{q}}^{O}-\omega_{o}}+\frac{f_{\boldsymbol{p}-\boldsymbol{q}}+N_{\boldsymbol{q}}}{\bar{\varepsilon}_{\boldsymbol{p}}^{o}-\bar{\varepsilon}_{\boldsymbol{p}-\boldsymbol{q}}^{o}+\omega_{o}}\right)=0
$$

For weak electron-optical phonon interactions, where $N_{\boldsymbol{q}} \ll 1$ and $f_{\boldsymbol{p}} \ll 1$, we obtain

$$
\bar{\varepsilon}_{\boldsymbol{p}}-\varepsilon_{\boldsymbol{p}}=\Delta \varepsilon_{\boldsymbol{p}}=P \sum_{\boldsymbol{q}} C_{\boldsymbol{q}}^{2}\left|M\left(q_{z}\right)\right|^{2} \frac{1}{\bar{\varepsilon}_{\boldsymbol{p}}^{o}-\bar{\varepsilon}_{\boldsymbol{p}-\boldsymbol{q}}^{o}-\omega_{o}}
$$




\section{Calculation}

Using the expression in Eqn.(37) we calculate the change in the ground state energy of the polaron. Changing the summation over $\boldsymbol{q}$ to integration, using cylindrical polar coordinates, and making the following change of variables,

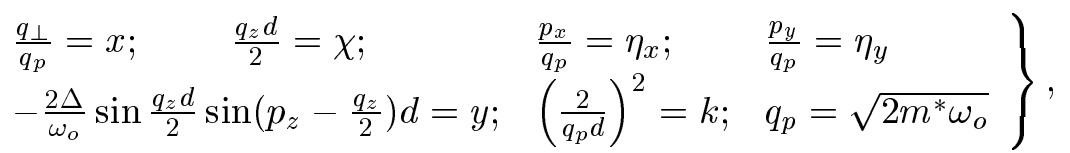

the shift in ground state energy $\Delta \varepsilon_{\boldsymbol{p}}=\bar{\varepsilon}_{\boldsymbol{p}}-\varepsilon_{\boldsymbol{p}}$, then becomes

$$
\begin{aligned}
\Delta \varepsilon_{\boldsymbol{p}}= & -\frac{\alpha}{\pi^{2} d}\left(\frac{\omega_{o}}{2 m^{*}}\right)^{\frac{1}{2}} P \int_{-\frac{\pi}{2}}^{\frac{\pi}{2}}|m(\chi)|^{2} d \chi \int_{0}^{\infty} \frac{x d x}{k \chi^{2}+x^{2}} . \\
& \cdot \int_{0}^{2 \pi} \frac{d \theta}{1+y+x^{2}-2 x\left(\eta_{x} \cos \theta+\eta_{y} \sin \theta\right)}
\end{aligned}
$$

On evaluation of the integrals with respect to $\theta$ and $x$, one finds

$$
\begin{aligned}
\Delta \varepsilon_{\boldsymbol{p}}= & -\frac{\alpha}{\pi d}\left(\frac{\omega_{o}}{2 m^{*}}\right)^{\frac{1}{2}} P \int_{-\frac{\pi}{2}}^{\frac{\pi}{2}} \frac{|m(\chi)|^{2} d \chi}{\sqrt{\left(y+1-k \chi^{2}\right)^{2}+4 k \chi^{2}\left(\eta_{x}^{2}+\eta_{y}^{2}\right)}} \\
& \cdot \ln \left[\frac{\sqrt{\left(y+1-k \chi^{2}\right)^{2}+4 k \chi^{2}\left(\eta_{x}^{2}+\eta_{y}^{2}\right)} \cdot(y+1)+(y+1)^{2}-k \chi^{2}\left(1+y-2\left(\eta_{x}^{2}+\eta_{y}^{2}\right)\right)}{k \chi^{2}\left\{\sqrt{\left(y+1-k \chi^{2}\right)^{2}+4 k \chi^{2}\left(\eta_{x}^{2}+\eta_{y}^{2}\right)}+y+1-k \chi^{2}-2\left(\eta_{x}^{2}+\eta_{y}^{2}\right)\right\}}\right]
\end{aligned}
$$

At low temperatures, when electron-optical phonon interactions result in polaron effects, the electron momentum is less than that of the phonon, i.e. $\boldsymbol{p}<\boldsymbol{q}$. This implies that

$$
\left|\frac{k \chi^{2}\left(\eta_{x}^{2}+\eta_{y}^{2}\right)}{\left(y+1-k \chi^{2}\right)^{2}}\right| \ll 1
$$

Therefore, the expression $\frac{1}{\sqrt{\left(y+1-k \chi^{2}\right)^{2}+4 k \chi^{2}\left(\eta_{x}^{2}+\eta_{y}^{2}\right)}}$ in Eqn.(40) can be expanded in Binomial series, retaining only first and lower-order terms, i.e.

$$
\frac{1}{\sqrt{\left(y+1-k \chi^{2}\right)^{2}+4 k \chi^{2}\left(\eta_{x}^{2}+\eta_{y}^{2}\right)}}=\simeq \frac{1}{y+1-k \chi^{2}}\left[1-\frac{2 k \chi^{2}\left(\eta_{x}^{2}+\eta_{y}^{2}\right)}{\left(y+1-k \chi^{2}\right)^{2}}\right]
$$

Using this approximation in Eqn.(40), with

$$
a=k \chi^{2}, \text { and } b=y+1,
$$

$\Delta \varepsilon_{\boldsymbol{p}}$ is found to be

$$
\begin{aligned}
\bar{\varepsilon}_{\boldsymbol{p}}-\varepsilon_{\boldsymbol{p}} & =-\frac{p_{x}^{2}+p_{y}^{2}}{2 m^{*}}\left(\frac{Y_{1}}{\pi q_{p} d}\right) \alpha-\left(\frac{Y_{2}}{\pi q_{p} d}\right) \alpha \omega_{o} \\
& =-\frac{p_{\perp}^{2}}{2 m^{*}} \beta_{1}(d) \alpha-\beta_{2}(d) \alpha \omega_{o}
\end{aligned}
$$


where

$$
\begin{aligned}
Y_{1} & =P \int_{-\frac{\pi}{2}}^{\frac{\pi}{2}}|m(\chi)|^{2} d \chi\left(\frac{a+b}{(a-b)^{2} b}-\frac{2 a}{(a-b)^{3}} \ln \frac{a}{b}\right) \\
Y_{2} & =P \int_{-\frac{\pi}{2}}^{\frac{\pi}{2}}|m(\chi)|^{2} d \chi \frac{1}{a-b} \ln \frac{a}{b} \\
\beta_{1}(d) & =\frac{Y_{1}}{\pi q_{p} d} \\
\beta_{2}(d) & =\frac{Y_{2}}{\pi q_{p} d}
\end{aligned}
$$

The effective mass $m_{\text {pol }}^{*}$ of the polaron in the planes perpendicular to the SL axis is the reciprocal of the second derivative of its energy with respect to the component of momentum in the planes perpendicular to the SL axis. Thus

$$
\begin{aligned}
\frac{1}{m_{\text {pol }}^{*}} & =\frac{\partial^{2} \bar{\varepsilon}_{\boldsymbol{p}}}{\partial p_{\perp}^{2}}=\frac{1}{m^{*}}\left(1-\alpha \beta_{1}(d)\right) \\
\text { or } \quad m_{\text {pol }}^{*} & =m^{*}\left(1-\alpha \beta_{1}(d)\right)^{-1}
\end{aligned}
$$

and for $\alpha \ll 1$,

$$
m_{\text {pol }}^{*}=m^{*}\left(1+\alpha \beta_{1}(d)\right)
$$

\section{Results And Discussions}

It is observed from Eqn.(44) that the shift in energy, as a result of the formation of polaron, depends on the integrals $Y_{1}$ and $Y_{2}$. The effective mass of the polaron also depends on $Y_{1}$ only. Both $Y_{1}$ and $Y_{2}$ depend on the matrix element of the potential trough or well which appears as a result of the electron-phonon interaction. Hence one must know the form of the potential in order to evaluate these integrals. In the case of an infinite square well potential $\left(U_{o} \rightarrow \infty\right)$, the model proposed by Shik[30] can be used to show that the matrix element $|m(\chi)|$ of the infinite square well potential is

$$
|m(\chi)|^{2}=\frac{\sin ^{2} \chi}{\chi^{2}}
$$

With this form of the matrix element, $Y_{1}$ and $Y_{2}$ are not analytically integrable, therefore these integrals were evaluated numerically.

Assuming that $\omega_{o}=10^{14} \mathrm{~s}^{-1}, m^{*}=8.0 \times 10^{-31} \mathrm{~kg}, p_{\perp}=10^{-9} \mathrm{kgms}^{-1}, \hbar=1.05 \times 10^{-34} \mathrm{Js}$, and considering that for slow electrons $(\boldsymbol{p}<\boldsymbol{q}), p_{z}$ can be assumed to be negligible, the shift in energy $\Delta \varepsilon_{\boldsymbol{p}}$ was obtained as a function of $d$ and $\Delta$. Typical values of $d$ and $\Delta$ are in the order of $10^{-8} \mathrm{~m}$ (few hundreds of $\AA$ ) and $10^{-19} \mathrm{~J}$ respectively. Typical values of $\alpha$ for III-V compounds lie within 0.015-0.080, and for II-VII compounds from 0.39-0.65 [32]. These limits of the $\alpha$ values for these semiconductor compounds were used in all our calculations. In particular, $d=10^{-8} \mathrm{~m}$, $\Delta=0.5 \times 1.6 \times 10^{-19} \mathrm{~J}$ and $\alpha=0.39$ yielded $\Delta \varepsilon_{\boldsymbol{p}} / \hbar \omega_{o}=-0.070$ or $\Delta \varepsilon_{\boldsymbol{p}}=-0.0046 \mathrm{eV}$. 
In figure $1, \Delta \varepsilon_{\boldsymbol{p}} / \hbar \omega_{o}$ was plotted against $d$ for $\alpha=0.015,0.080,0.39,0.65$ and $\Delta=0.5 \mathrm{eV}$. Straight lines which become steeper as $\alpha$ increases were obtained. This means that the shift in the energy decreases (more negative) with increasing electron-phonon coupling constant. If the coupling constant is fixed at 0.39 , and $\Delta \varepsilon_{\boldsymbol{p}} / \hbar \omega_{o}$ plotted against $d$ for $\Delta=0.05,0.5,1.0$ and $2.0 \mathrm{eV}$ (figure 2), the lines become less steeper as $\Delta$ increases. Thus the shift in energy increases (becomes more positive) with increasing bandwidth.

$\Delta \varepsilon_{\boldsymbol{p}} / \hbar \omega_{o}$ was plotted against $\Delta$ for $\alpha=0.015,0.080,0.39,0.65$ and $d=200 \AA$. The curves obtained are shown in figure 3. First, we observed that the curves shift down the $\Delta \varepsilon_{\boldsymbol{p}} / \hbar \omega_{o}$ axis as $\alpha$ was increased. This again confirms the observation made in figure 1 that $\Delta \varepsilon_{\boldsymbol{p}}$ decreases with increasing coupling constant. Secondly, we observed that for small $\alpha(\alpha=0.015) \Delta \varepsilon_{\boldsymbol{p}} / \hbar \omega_{o}$ varies slowly and is almost constant for large values of $\Delta$. If $\alpha$ is fixed at $\alpha=0.39$ and $\Delta \varepsilon_{p} / \hbar \omega_{o}$ plotted against $\Delta$ for $d=200,500,700$ and $1000 \AA$, the curves, as observed in figure 4 , shift down the $\Delta \varepsilon_{\boldsymbol{p}} / \hbar \omega_{o}$ axis as $d$ increases. Thus $\Delta \varepsilon_{\boldsymbol{p}}$ depends on the SL parameters $d$ and $\Delta$.

The surface plot shown in figure 5 is a plot of $\Delta \varepsilon_{\boldsymbol{p}}(d, \Delta) / \hbar \omega_{o}$ against $(d, \Delta)$ for $\alpha=0.39$. This plot and similar plots for $\alpha=0.015,0.080$ and 0.65 (not shown) indicate that the shift in energy decreases with increasing $\alpha$ or increasing $d$, but increases with increasing $\Delta$, summarising the observations made in figures 1 to 4 .

Surface plots of the effective polaron mass $m_{\text {pol }}^{*}(d, \Delta)$ against $(d, \Delta)$ for $\alpha=0.015,0.080$, 0.39 and 0.65 gave surfaces through 1 and parallel to the $d-\Delta$ plane. Thus, the effective mass varies very little with the coupling constant $\alpha$ and SL parameters, $d$ and $\Delta$; and the ratio of the polaron effective mass to electron mass is almost 1 for all values of $\alpha, d$ and $\Delta$. To have an idea of the smallness of the difference between the polaron effective mass and the electron mass, the ratio of the polaron effective mass to electron mass was evaluated, assuming $\alpha=0.65, d=100 \AA$ and $\Delta=0.5 \mathrm{eV}$. The result obtained is

$$
\frac{m_{p o l}^{*}}{m^{*}}=1+1.96657 \times 10^{-33}
$$

which is approximately equal to 1 , so that $m_{\text {pol }}^{*} \simeq m^{*}$. Thus the polaron effective mass along the plane perpendicular to the SL axis is approximately equal to electron mass.

\section{Conclusion}

The shift $\Delta \varepsilon_{\boldsymbol{p}}$ in the ground state energy of an electron whose interaction with a phonon results in the formation of a polaron is dependent on the SL parameters $d$ and $\Delta$, and the coupling constant $\alpha$, if the potential well in which the electron is trapped is assumed to be an infinite square well. The shift in the ground state energy decreases with increasing $d$ and $\alpha$, but increases with increasing $\Delta$. For small values of $\alpha$, the shift in the ground state energy as a function of $\Delta$ varies slowly with $\Delta$, and is approximately a constant for large $\Delta$. 
The effective mass along the planes perpendicular to the SL axis was found to be approximately equal to the electron mass for all typical values of $d, \Delta$ and $\alpha$, the difference between the effective mass and the electron mass being of the order $10^{-33}$.

\section{Acknowledgements}

This work was done within the framework of the Associateship Scheme of the Abdus Salam International Centre for Theoretical Physics, Trieste Italy. Financial support from the Swedish International Development Cooperation Agency is acknowledged.

\section{References}

[1] T. K. Mitra, A Chatterjee, and S. Mukhopadhyay, Phys. Rep. 153, 91 (1987).

[2] F. M. Peeters and J. T. Devreese, in Solid State Physics, edited by F. Seitz and D. Turnbull (Academic, New York, 1984), Vol. 38, p81.

[3] T. D. Lee, F. E. Low, and D. Pines, Phys. Rev. 90, 297 (1953).

[4] D. L. Lin, R. Chen, and T. F. George, J. Phys. Condens. Matter 3, 4645 (1991).

[5] G. Q. Hai, F. M. Peeters, and J.T. Devreese, Phys. Rev. B 48, 4666 (1993).

[6] G. Q. Hai, F. M. Peeters, and J.T. Devreese, Phys. Rev. B 47, 10358 (1993).

[7] Chuan-Yu Chen, Shi-Dong Liang, and Ming Li, J. Phys. Condens. Matter 6, 1903 (1994).

[8] Jun-Jie Shi, Xiu-qin Zhu, Zi-xin Liu, Shao-hua Pan, and Xing-yi Li, Phys. Rev. B 55, 4670 (1997).

[9] L. Wendler, A. V. Chaplik, R. Haupt, and O. Hipólito, J. Phys. Condens. Matter 5, 8031 (1993).

[10] Hai-Yang Zhou and Shi-Wei Gu, Solid State Commun. 88, 291 (1993).

[11] Feng-Qi Zhao, Xu Wang, and Xi-Xia Liang, Phys. Lett. A 175, 225 (1993).

[12] R. Haupt and L. Wendler, Solid State Commun. 89, 741 (1994).

[13] Bao-hua Wei, K. W. Yu, Fa Ou, J. Phys. Condens. Matter 6, 1893 (1994).

[14] Bao-hua Wei and K. W. Yu, J. Phys. Condens. Matter 7, 1059 (1995).

[15] J. J. Licari, Solid State Commun. 29, 625 (1979).

[16] X. X. Liang, S. W. Gu, and D. L. Lin, Phys. Rev. B 34, 2807 (1986). 
[17] Bao-hua Wei, Xun-jie Zhao, and Shi-wei Gu, Phys. Rev. B 41, 1368 (1990).

[18] Tian-Quan Lu and Jin-Song Li, J. Phys. Condens. Matter 5, 3365 (1993).

[19] M. H. Degani and O. Hipólito, Surf. Sci. 196, 459 (1988).

[20] S. Ban, X. X. Liang, and R. Zheng, Phys. Rev. B 51, 2351 (1995).

[21] G. A. Farias, M. H. Degani, and O. Hipólito, Phys. Rev. B 43, 4113 (1991)

[22] E. P. Pokatilov, S. I. Beril, V. M. Fomin, and G. Yu. Riabukhin, Phys. Status Solidi B 156, 225 (1989).

[23] A. Thilagam and J. Singh, Phys. Rev. B 49, 13583 (1994)

[24] R. Zheng, S. Ban, and X. X. Liang, Phys. Rev. B 49, 1796 (1994).

[25] C. Y. Chen, D. L. Lin, P.W. Jin, S. Q. Zhang, and R. Chen, Phys. Rev. B 49, 13680 (1994).

[26] A. Elangovan and K. Navaneethakrishnan, J. Phys. Condens. Matter 5, 4021 (1993).

[27] Hai-Yang Zhou and Shi-Wei Gu, Solid State Commun. 89, 937 (1994).

[28] A. Elangovan D. Shyamala, and K. Navaneethakrishnan, Solid State Commun. 89, 869 (1994).

[29] G. M. Shmelev, I. A. Chaikovskii, V. V. Pavlovich and E. M. Epshetein, Phy. Stat. Sol. 6 86697 (1977).

[30] A. Y. Shik, Fiz Tekh Poluprov. 7, 261 (1973).

[31] D. N. Zubarev, Soviet Physics Uspekhi, 71, 320 (1960).

[32] A. Anselm, in Introduction to Semiconductor Theory, p333 (English translation, Mir Publishers, 1981. Revised from the Russian the 1978 edition). 